\title{
Attosecond Metrology and Spectroscopy
}

\author{
Martin Schultze and Reinhard Kienberger \\ Ludwig-Maximilians-Universität and Technische Universität München
}

Germany

\section{Introduction}

Time resolved investigations of electron dynamics in a variety of sample systems as atoms, molecules and solids establish the spearhead of modern ultrafast science. The technology that enables scientists to track and control these processes is referred to as "attosecond metrology" and is associated in symbiosis with ultrashort laser source development.

The origin of attosecond metrology is linked to the advent of ultrashort mode locked laser sources - femtosecond lasers, chirped pulse amplification, carrier-envelope-phase stabilization - and the technological aspects of this development that rendered experiments with a temporal resolution of tens of attoseconds possible. The high-order harmonicgeneration process yields laser pulses in the wavelength region around $10 \mathrm{~nm}$ with durations below 100 attoseconds, at the same time being the shortest controllable signals. We introduce the different spectroscopic techniques that make use of this unprecedented temporal resolution. While the "attosecond transient recorder" constitutes an oscilloscope for electromagnetic fields oscillating at visible frequencies, "attosecond tunneling spectroscopy" as well as "attosecond transient absorption" provides real-time insight into the dynamic processes of electronic rearrangements in matter.

Looking at the evolution of the development of ultrashort pulses, it is remarkable that optical communication stood behind RF communication and electronics for more than a century. Light did not have to be "invented", it was there - due to our ability to see electromagnetic radiation at wavelengths between 380 and $480 \mathrm{~nm}$. Especially since man was able to control fire, light was used as a signal for information transport over wide distances: lighthouses, smoke signals, nautic flags; using optical telegraphy with movable signal arms, the 225 kilometers between Metz and Mainz could be covered in a few minutes. Just after the invention of the light bulb by Thomas Alva Edison and Heinrich Göbel, in 1886 Heinrich Hertz proved the radio waves which had been postulated by James Clerk Maxwell in 1864. Since switching rates for light bulbs were considerably smaller than the ones for radio waves, and since there were no transmission systems for light with low losses, within shor time signal communication via electric cables and RF signals was established. The spectacular advances in the development of optical fibers with extremely low losses (Nobel prize 2009 for Charles Kao) as well as the development of semiconductor-based incoherent and coherent light sources (LEDs and diode lasers) lead to the comeback and triumph of optical communication at the end of the laser century. These sources made possible to generate sufficiently short pulses to achieve extremely high switching and transmission rates. 
The needs in optical communications have motivated the development of light sources with short and ultrashort pulse duration significantly. Scientists from atomic, molecular and optical physics made significant contributions and profited from this development. After breaking the picosecond border in pulse duration, an own filed, ultrafast physics evolved. Light pulses of ultrashort duration are a key tool to investigate processes in microcosm. Ahmed Zewail was awarded the Nobel prize 1999 for femtosecond chemistry (Anon 1994). The laws of quantum optics dictate the duration at which processes in atoms, molecules and solids take place (Ferenc Krausz 2009). Electronic transitions in atoms, for example, can happen on a timescale of femtoseconds or attoseconds, where an attosecond (10-18 s) compares to one second approximately like a second to the age of the universe.

From photography we know that an image of a moving object can only be unblurred if the exposure time is significantly shorter than the duration of the movement. The timeresolution of the electronic processes mentioned above therefore needs pulse durations of less than one femtosecond. That's not so easy, since nature does not give us anything for free: Electromagnetic pulses, hence light pulses consist of many waves of different wavelengths which add up in a way that a temporarily confined signal - the pulse - is formed. The envelope within which the electric field oscillates with the carrier frequency defines the duration of the pulse. In order that a pulse can propagate in space, the carrier has to undergo at least one oscillation cycle, which sets a lower limit for the pulse duration. At $750 \mathrm{~nm}$, which is a common wavelength for ultrafast lasers, one oscillation cycle lasts about $2.5 \mathrm{fs}$. Amplified laser pulses at this central wavelength can be made as short as $3.3 \mathrm{fs}$, today (A. L. Cavalieri, E. Goulielmakis, et al. 2007). There is not much to be improved in terms of pulse duration at this wavelength. To achieve even shorter pulse durations, shorter wavelengths in the extreme ultraviolet (XUV) or x-ray regime are needed. Unfortunately, nature doesn't help us here, again. At these wavelengths no materials exist that could be used as laser media, directly. Besides demanding and expensive methods like free-electronlasers (FEL), which are big facilities with hundreds of meters extension, coherent light can be produced by a non-linear wavelength conversion from laser pulses in the visible range. The generation of high-order harmonics (McPherson et al. 1987) (Ferray et al. 1988) provides the possibility to produce attosecond pulses (Paul et al. 2001) (Christov et al. 1997). Before we concentrate on this method, let's have a look at the formation of ultrashort pulses comprising barely more than one oscillation cycle of the driver wave.

\section{State-of-the-art}

Laser pulses can be very short if their spectrum is extremely broad. At the high frequency of visible light in the range of Petahertz, it is sufficient to have a spectral width of a few Terahertz to achieve pulse durations of less than one picosecond. This can be realized at very different ways. Very common are quality switched (Q-switched) lasers, which are equipped with a device in the laser resonator that influences - actively or passively - the finesse of the resonator and therefore generates pulsed radiation. In order to achieve even shorter pulses, a number of technological approaches have been realized, making different wavelengths in the laser resonator oscillate synchronously. If the oscillations of the different longitudinal resonator modes, that are supported by the gain medium, have a constant phase relation (i.e. mode locking), a short pulse is created. The more resonator modes couple, the shorter is the pulse. 
After dye lasers had dominated short pulse technology, today nearly all practically all short pulse lasers are based on solids, i.e. glasses and crystals, as the gain medium. In the visible and neighboring infrared range, sapphire doped with titanium ions has prevailed. Ti:Sa lasers have two main advantages - besides being relatively easy to handle: They can be pumped by commercially available, frequency doubled infrared lasers and have a comparably broad amplification bandwidth. To make use of this bandwidth as many modes in the resonator as possible have to be mode-locked and the propagation of a short pulse has to be favored with respect to continuous radiation. Aside from optical components that compensate for dispersion in the resonator, this is mainly made possible by saturable absorbers or concepts based on the optical Kerr effect.

Saturable absorbers are semiconductors with the special characteristic to escalate reflectivity when incoming radiation exceeds a certain threshold intensity. Such a component improves the finesse of a resonator selectively for short pulses and enables mode locking. Another common technology, which can be combined with saturable absorbers, is Kerr-lens modelocking which makes use of the intensity dependence of the refractive index (Kerr-effect) of certain materials. The shorter, i.e. intenser, an incoming pulse is in the material, the stronger self-focusing it experiences. In combination with an aperture such a material ensures that short pulses will be amplified preferably. In combination with chirped mirrors that have been invented in 1990 (Szipocs et al. 1994) it was possible to demonstrate pulse durations shorter than $10 \mathrm{fs}$ with this method. Chirped mirrors (see Figure 2,3) are coated in a way that different wavelengths penetrated to a differing depth of the layers. The word "chirp" refers to the fact that frequencies - like in the singing of birds - are changing instantaneously. An incoming light pulse whose spectral components are asynchron (chirped) can be compressed to the minimum pulse duration which is supported by its spectrum. Using techniques of this kind, today it's possible to build oscillators whose optical spectrum comprises nearly a full octave, so that the high frequency part of the spectrum oscillates twice that fast as the low frequency one. In the example discussed here, the spectrum reaches from 450 to $1050 \mathrm{~nm}$, supporting a pulse duration of about $5 \mathrm{fs}$. The ability to generate ultra-short laser pulses is not the only aspect that puts mode locked lasers in the focus of science. The lasers resonator emits pulses with a repetition rate corresponding to the inverse round trip time in the cavity. This repetition rate therefore depends on the length of the laser cavity and typical values are on the order of $100 \mathrm{MHz}$. It has to be emphasized that this periodicity in time translates to a periodicity in the Fourier domain: The spectrum of the pulse train emitted by a mode locked oscillator is not continuous but consists of perfectly equidistant spectral lines. This phenomenon was dubbed "frequency comb" and in 2005 Theodor Hänsch was awarded the Nobel Prize for the proposal to use this unique feature for frequency resolved spectroscopy (Reichert 1999) (Telle et al. 1999). Nowadays it is the basis for uncountable applications in the high resolution spectroscopy given that the spacing of the comb lines for a standard mode locked oscillator emitting at $800 \mathrm{~nm}$ with a repetition rate of $100 \mathrm{MHz}$ is only 0.00021 nanometers.

Simultaneously the frequency comb found an important application also in the synthesis of ultrashort laser pulses themself. Relying on the measurement of difference frequencies between the individual comb lines on can ensure that every laser pulse emitted has an identical evolution of the electric field underneath the intensity envelope, the so-called "waveform". Figure 1 highlights the importance of this technology for ultrashort laser pulses containing only few optical cycles. 


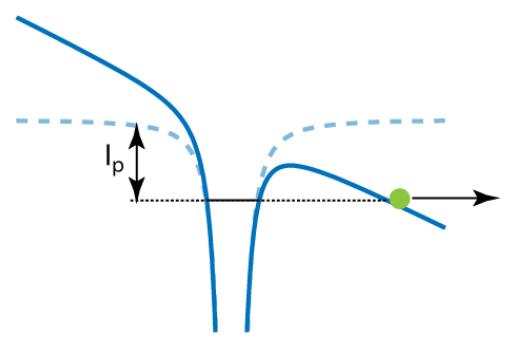

step 1) tunnel ionization

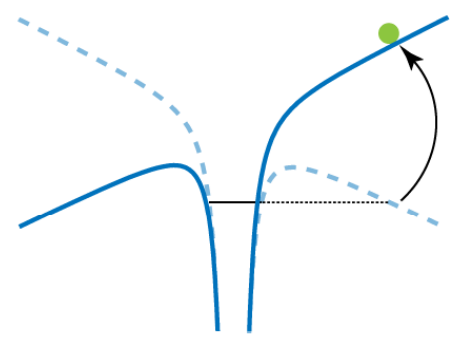

step 2) acceleration in the laser electric field

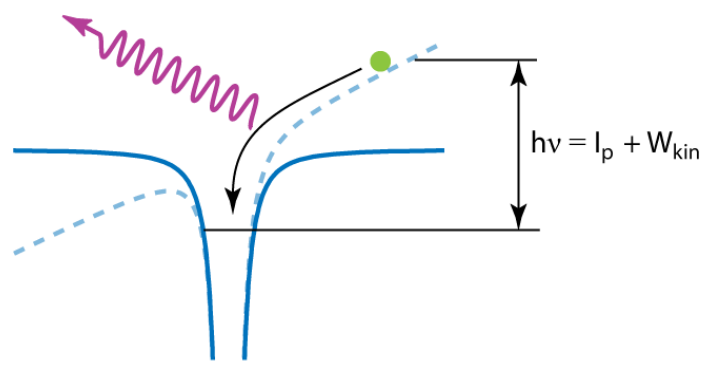

step 3) recombination and photon emission

Fig. 1. High harmonic generation (HHG)

Ultra intense laser pulses can ionize matter but the released electrons not necessarily leave the atom forever. The "3-step-model" invented by Corkum in 1993 describes this process. If the field strength of the laser pulses is strong enough to suppress the coulomb potential binding the electron to the core, the electrons can tunnel out of the atom (step 1). This free electron is then accelerated in the laser electric field (step 2) that reverts its sign once per period and thus forces the electron to return to its parent ion with a non-vanishing probability and recombine there under the emission of radiation. This process is unlikely but not impossible; the conversion efficiencies reported are about $10^{-7}$. Still this technique offers amazing possibilities to the experimentalist due to the unique time structure of the emitted pulses (counted in attoseconds) and the high photon energies that can be achieved (up to $\mathrm{keV}$ photons are demonstrated). 
Modern mode locked oscillators achieve Nano-Joule pulse energies which in turn can be focused to intensities in the range of $\sim 1011 \mathrm{~W} / \mathrm{cm}^{2}$ insufficient to cause nonlinear response of even ionization of matter (effective ionization of rare gases sets in at laser intensities around $\left.10^{14} \mathrm{~W} / \mathrm{cm}^{2}\right)$.

Pushing ultrafast technology towards intensities above this threshold requires additional efforts (Figure 2). Usually mode locked oscillators are followed by amplification stages that work according to the "chirped-pulse-amplification" scheme (STRICKLAND \& MOUROU 1985). The individual spectral components are dispersed and thus the laser pulses are stretched in time leading to drastically reduced peak intensity while maintaining the coherence properties. Subsequently the pulses are amplified in a second laser active medium (crystal or fiber). For this purpose the repetition rate of the oscillator pulse train has to be reduced drastically since the amplifying medium has to fully absorb the thermal energy that goes along with the million fold amplification of the pulses. The difficult handling of the increasing average power and the lack of high-repetitive pump lasers currently render repetition rates higher than a few kilohertz impossible.

For selection of the individual pulses out of the high-repetitive oscillator pulse train contemporary laser systems rely on "pulse-picking" arrangements employing the electro optic effect in a Pockels-cell between orthogonal polarizing filters. After the amplification process (see Fig. 2) the timing of the individual wavelength components is rectified to achieve a short laser pulse by grating- or prism compressors frequently in combination with chirped mirrors.

Unfortunately the amplification process not only reduces the repetition rate but - due to the natural gain window of the laser active medium- also the spectral bandwidth of the laser pulses. To achieve near-single cycle pulses the amplification has to be accompanied by a system that coherently generates additional frequency components on either side of the spectrum. The commonly used systems to achieve that are based on a nonlinear process that was dubbed "self-phase-modulation". The nonlinear interaction of the electric field of energetic laser pulses with rare gases can yield spectra spanning form 450 to $1100 \mathrm{~nm}$ covering the whole range of human vision (cp. Fig. 3). By the use of additional chirped mirrors to once again synchronize the individual spectral components state-of-the-art ultrashort pulse laser systems achieve mJ pulse energies within less than 3,5 femtoseconds (A. L. Cavalieri, E. Goulielmakis, et al. 2007) duration. Well focused, no matter can withstand the strength of the electric field that oscillates only 1,5 times during such a pulse reaching intensities up to $1018 \mathrm{~W} / \mathrm{cm}^{2}$.

If such pulses are used for the generation of high-order harmonics, as mentioned before (see also Fig. 1), one has to distinguish between pulses with many cycles of the driver wave and few-cycle pulses. In the first case, a train of attosecond pulses is generated, which requests that the the sub-cycle electron-light interaction is repeated over several oscillation cycles under exactly the same conditions. Only then, no information is lost during the accumulation of attosecond signals from different cycles of the laser pulse, since the characteristics of the interaction products (e.g. the properties of photoelectrons) are the same. The same request has to be fulfilled when a train of attosecond pulses being separated by half-cycle of the driving laser field is used in a cross correlation with the driving field itself. Even if the laser field had constant amplitude over many cycles, metrology would be restricted to processes being shorter than one half-cycle of the laser field. 


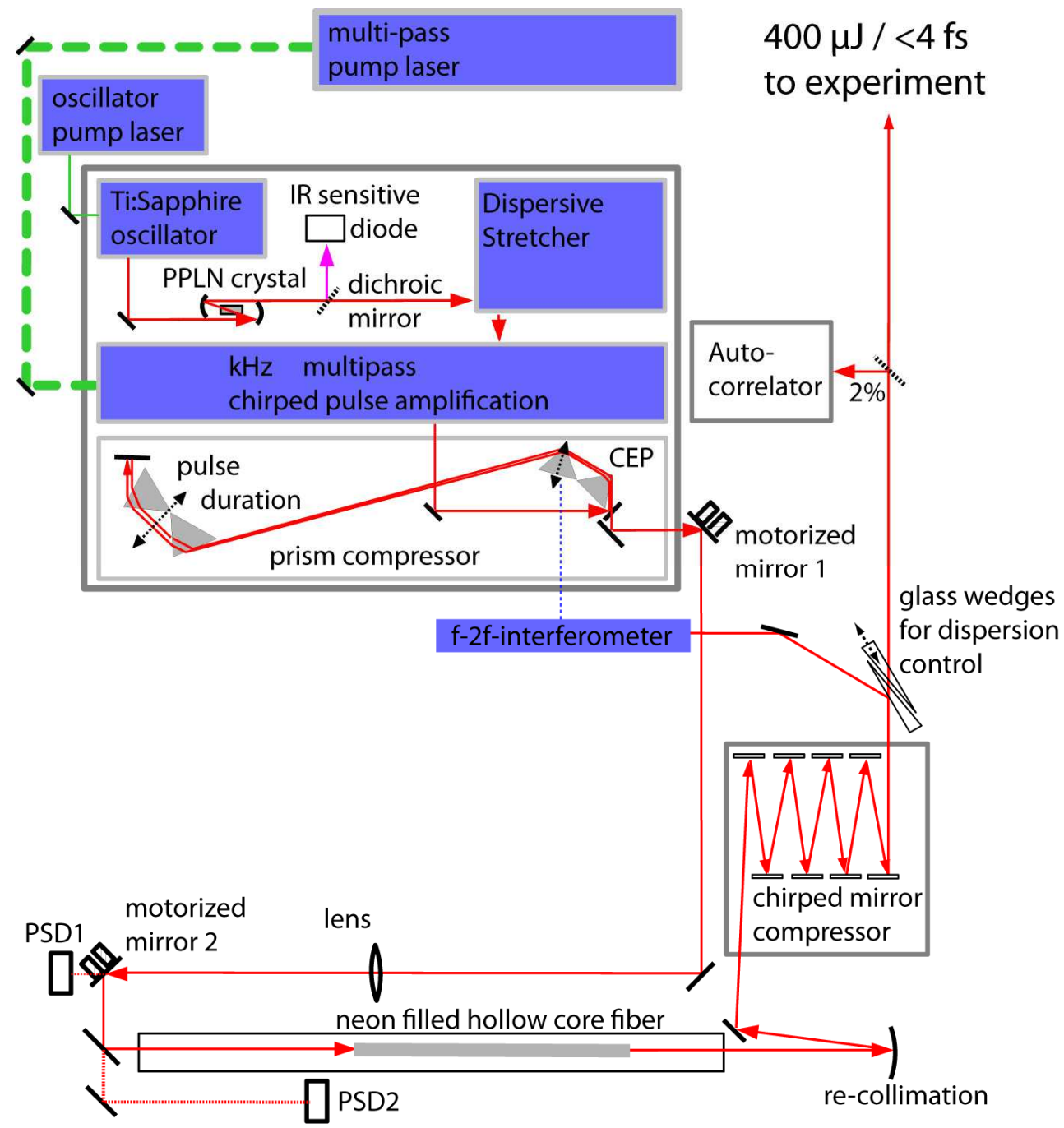

Fig. 2. Ultrafast Laser

A continuously pumped mode-locked oscillator emits broadband pulses of fewfemtosecond duration. These pulses are dispersed in time up to ps duration to reduce their peak-intensity and subsequently amplified in a multi-pass amplifier pumped by a multi-mJ Q-switch laser usually operating with $\mathrm{kHz}$ repetition rates. Later a prism-compressor rectifies the timing of the different wavelength components reducing the pulse duration again to 10s of femtoseconds. The nonlinear process of self-phase-modulation that takes place in a gas filled hollow-core fiber adds additional spectral components on either side of the amplified spectrum and a set of chirped mirrors converts the octave spanning output spectrum (cp. Figure 3) into ultrashort visible light pulses. 

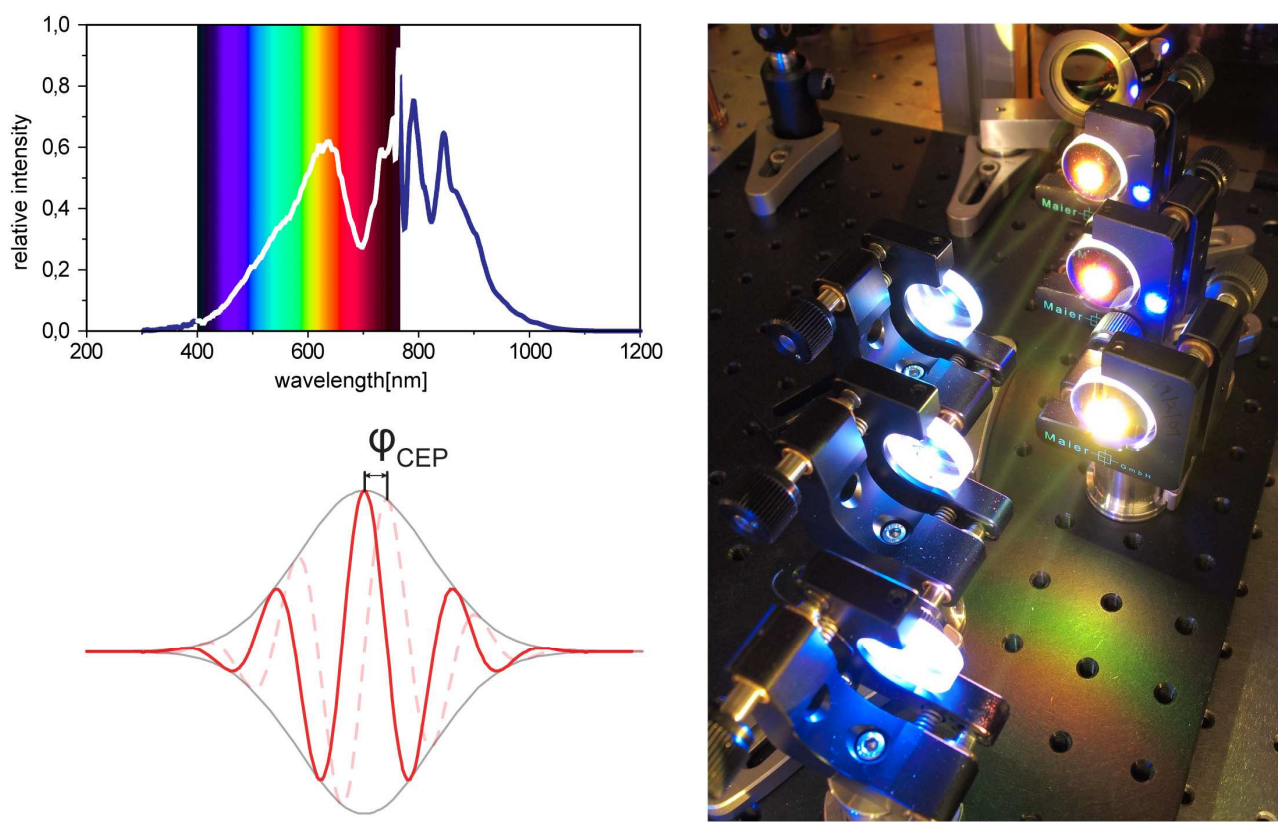

Fig. 3. Ultrashort Laser pulses

As a consequence of the uncertainty relation short laser pulses require an extended spectrum. To synthesize laser pulses consisting of little more than one cycle of the electric field the spectrum has to span an octave (it has to coherently contain frequencies between $v$ and $2 \mathrm{v}$ ). Given the human eyes sensitivity being restricted to a range between $400 \mathrm{~nm}$ and $750 \mathrm{~nm}$, single cycle laser pulses appear white as seen on the photograph that shows the scattered light if such pulses are reflected off the multilayer mirrors mentioned in the text. The graph compares the measured spectrum of such pulses with the sensitivity range of human sight (rainbow shaded area). The plot below highlights how for such short pulses the phase (carrier-envelope-phase CEP) between the intensity envelope function and the oscillations of the carrier wave defines the waveform and thus the action of the pulse in nonlinear processes.

Multi-cycle attosecond metrology (Ferenc Krausz 2009) can be used to investigate periodically-repeated recollisions which are driven by the multi-cycle light wave and trigger the process under study in a correlated manner in the same interaction within each wave cycle. One of the two correlated processes can serve as a clock for the other. Inventing this concept, (Niikura et al. 2002) demonstrated that the vibrational motion of a molecule triggered by ionization clocks the recollision electron and (Niikura et al. 2003) showed the case vice versa for a diatomic molecule.

In most laser systems, multi-cycle pulses have a Gaussian envelope which results in attosecond pulses with varying intensity and photon energy within the pulse train generated, If these pulses trigger even simple processes a retrieval of the process gets highly complicated. 
Therefore, employing few-cycle pulses for the synthesis of high-order-harmonics (Fig. 1) becomes highly significant. Given a smart choice of the phase between the intensity envelope and the oscillations of the carrier wave, waveforms can be generated where the intensity contrast between adjacent maxima of the electric field is maximized.

Since every single half cycle contributes to the emission of high-harmonics the highest intense half cycle apparently leads to the emission of the highest energetic photons. Filtering only this radiation out of the emitted spectrum thus yields coherent X-ray pulses with a duration corresponding to only a fraction of the driving lasers half-cycle duration. The shortest light bursts demonstrated ever have been generated in this manner: Lasting only about 80 attoseconds they contain roughly $10^{8}$ photons per pulse at a central wavelength of $12 \mathrm{~nm}$ deep in the ultraviolet.

As has been discussed, the parameters of the driving laser pulse are extremely important for the characteristics of the generated harmonic radiation. Pulse duration, pulse energy, photon energy, and the number of attosecond bursts (single pulses or a train) are given by the properties of the driving laser pulse. In terms of the photon energy, the wavelength of the driving pulse is crucial. It may be counterintuitive on first glance but longer wavelength driver pulses are able to generate harmonics at higher photon energy (Vladislav S. Yakovlev et al. 2007) since the electron can accumulate more energy during its trip to the continuum (in the semi classical picture). Therefore the development of few-cycle laser pulses in the infrared spectral range is pursued by several groups in the world.

(Fuji et al. 2006) have demonstrated an OPA system whose pulses comprise only a few oscillation cycles at $2.1 \mu \mathrm{m}$ carrier wavelength. IR-driven HHG was pioneered by L'Huillier and co-workers (L'Huillier \& Ph. Balcou 1993) and DiMauro and co-workers (Sheehy et al. 1999) followed by studies of (Bellini 2000), (Shan \& Chang 2001). As pointed out by DiMauro, the favourable scaling of the maximum photon energy with driver wavelength may open the way to generating coherent light and attosecond pulses at photon energies substantially beyond the kiloelectronvolt frontier, which was reached recently with NIR few-cycle light (J. Seres et al. 2005). This follows from the fact that the cutoff, i.e. the highest achievable energy in HHG (see Fig 1) scales with the ponderomotive potential - the cycle averaged quiver energy an electron gains in an oscillating field - and therefore with the square of the driving wavelength.

Another technique that aims at increasing the flux of HHG especially at high photon energies is the so-called quasi phase-matching (QPM). In this approach one tries to overcome a problem that limits the conversion efficiency between driver and harmonic radiation. Dispersion in the non-linear medium used for HHG results in a phase mismatch between the driver wave in the visible and NIR spectral range and the short wavelength product of the conversion process. This leads to a destructive interference after a certain distance and reduces the flux of the harmonic radiation. Methods like using a modulated fiber (Christov et al. 1998) as a cell for the conversion process or using several targets to modulate the density of the conversion medium aim at bringing the both waves involved in cadence again. Especially for photon energies in the $\mathrm{keV}$ range, where the phase mismatch plays a crucial role, this method is of great importance. 


\section{Experiments and applications}

If solids are used as a medium for the frequency upconversion to the XUV or x-ray regime, a different mechanism comes into play (Naumova et al. 2004); (Tsakiris et al. 2006); (Baeva et al. 2006)). At ultra-high intensities, the leading edge of a laser pulse can turn the solid surface into a high-density plasma where the electrons execute oscillations at the plasmavacuum interface driven by the ultra-intense field. The light pulse bounces back from the surface as if it were reflected by an oscillating mirror with relativistic speed (Bulanov et al. 1994) (Lichters et al. 1996); (Gordienko et al. 2004); (Tsakiris et al. 2006). Since the time it takes the mirror to sweep through a half period of the reflected field is contracted when the mirror and the incident wave counter-propagate, the reflected wave is blue-shifted. The highest occurring frequency is then given by the maximum Doppler upshift of $\Omega_{\max }$ $4 \gamma^{2} \max \omega_{\mathrm{L}}=4\left(1+\mathrm{a}_{0}^{2}\right) \omega_{\mathrm{L}}$, with $\gamma_{\max }$ being the relativistic factor and $\omega_{\mathrm{L}}$ and $\mathrm{a}_{0}$ being the frequency and vector potential of the laser pulse. The emission of high-frequency photons is confined to small fractions of the laser period resulting in a periodic train of high-energy photon bursts. In a few-cycle laser field the emission of the high-energy photons is confined to the central wave cycle. Spectral filtering may therefore lead to single attosecond pulse generation (Ferenc Krausz 2009).

After its advent at the beginning of the century attosecond science soon was recognized to have the potential to add unprecedented temporal resolution to most of the spectroscopic techniques that have been developed at large scale facilities and laboratories around the world. Particularly (photo-) electron spectroscopy (see Fig. 4) was enriched by the attosecond toolbox when attosecond streaking spectroscopy emerged (cp. Fig 5) (Hentschel et al. 2001) (Itatani et al. 2002) (R Kienberger et al. 2002) (Markus Kitzler et al. 2002) (R. Kienberger et al. 2004). Attosecond XUV pulses are focused onto the sample atoms (usually in the gas phase but also surface emission experiments have been demonstrated) causing the release of electrons with a kinetic energy corresponding to the energy difference between the XUV photon energy and the electron affinity. This kinetic energy is detected by measuring the electron arrival time on a detector placed in a known distance of the interaction volume as in common electron time of flight spectrometry. For this type of spectroscopy (in contrast to techniques involving trains of attosecond pulses) it is a prerequisite that the XUV pulse duration is significantly shorter than a quarter of the laser period. For lasers with a central wavelength on the red side of the visible spectrum that amounts to round 500 attoseconds and as a direct consequence of the uncertainty principle this goes along with a minimal spectral width of $3 \mathrm{eV}$ at a central photon energy around 100 $\mathrm{eV}$. Together with the spectral resolution around 1\% that is typically achieved in time of flight spectrometers this sets a lower limit to the spectral distance of features that shall be resolved and contrasts to the spectral resolution in the meV range that e.g. synchrotron sources achieve. Instead attosecond science exhibits its full strength in resolving ultrafast processes: For the sake of three orders of magnitude in spectral resolution it adds about 12 orders of magnitude in temporal resolution compared to the most contemporary XUV technologies known from synchrotrons and linear accelerators.

If the photoelectrons are set free in the presence of the electric field of a laser pulse their final kinetic energy can be altered (Hentschel et al. 2001) (Itatani et al. 2002). The net momentum change imparted on the electrons depends on the time delay between the attosecond XUV and the laser pulse (M. Schultze et al. 2011). A number of time of flight spectra is recorded with incrementally increased delay between the two pulses around the temporal coincidence. 


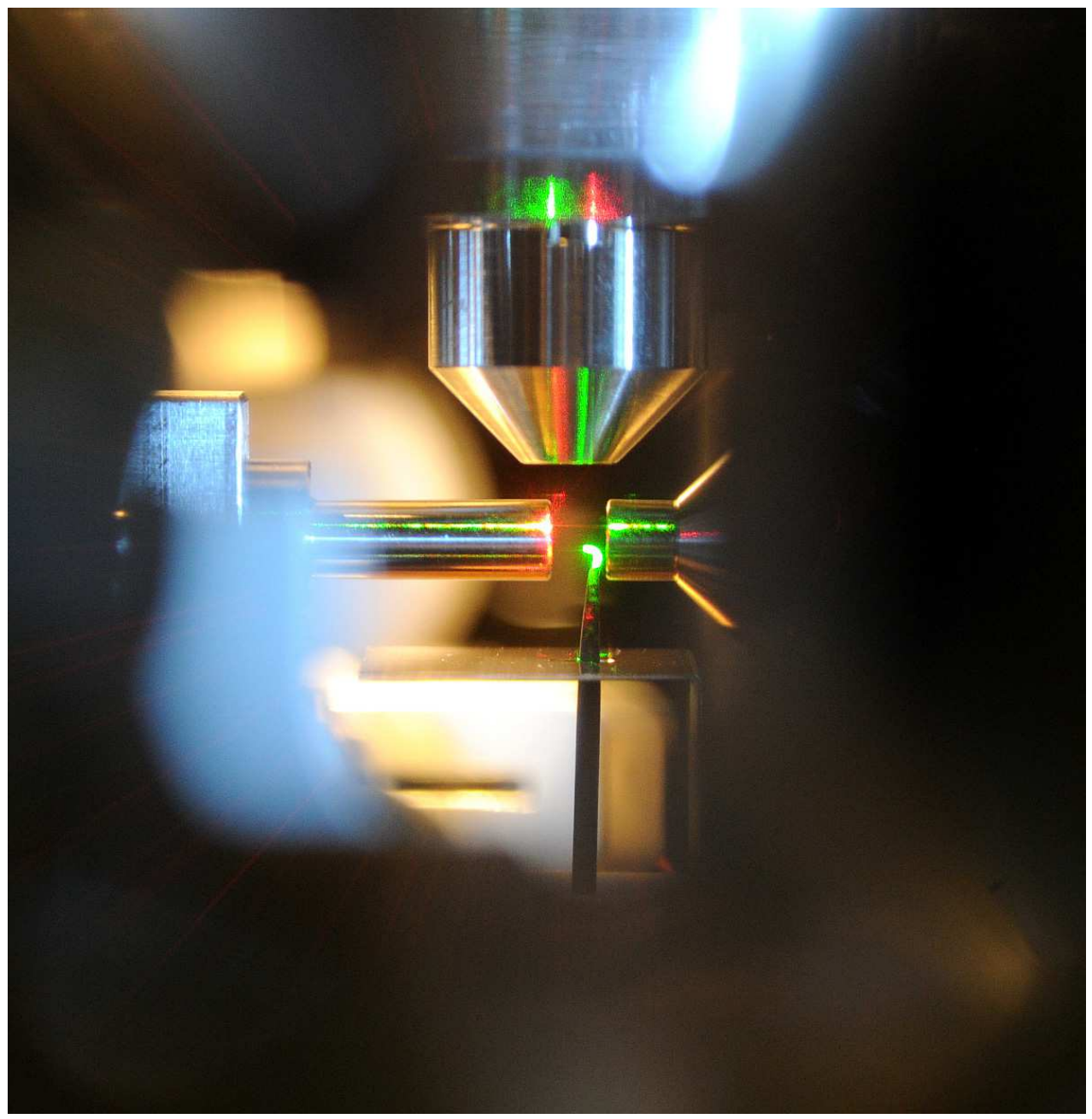

Fig. 4. Attosecond Spectroscopy

Experiments designed to make use of the unprecedented temporal resolution that ultrashort laser pulses can offer usually follow the "pump-probe-scheme“. A first laser pulse triggers the process under scrutiny and a second pulse probes the evolution after a defined time interval. From a sequence of a number of such measurements with variable temporal delay between the two pulses one can reconstruct the temporal evolution. The picture shows the inside of a vacuum chamber where such pump-probe experiments are performed based on the combination of visible laser pulses as pump- and attosecond XUV pulses as probe events. It is used to e.g. explore the electron dynamics in the atomic core. The gaseous sample streams out of the black nozzle into the interaction region and the released electrons are collected by a time-of-flight detector (cone from above) while the ionic fragments are detected by a mass spectrometer along the horizontal axis. The image was taken on the beam axis of the collinear propagating laser and xuv pulses . 


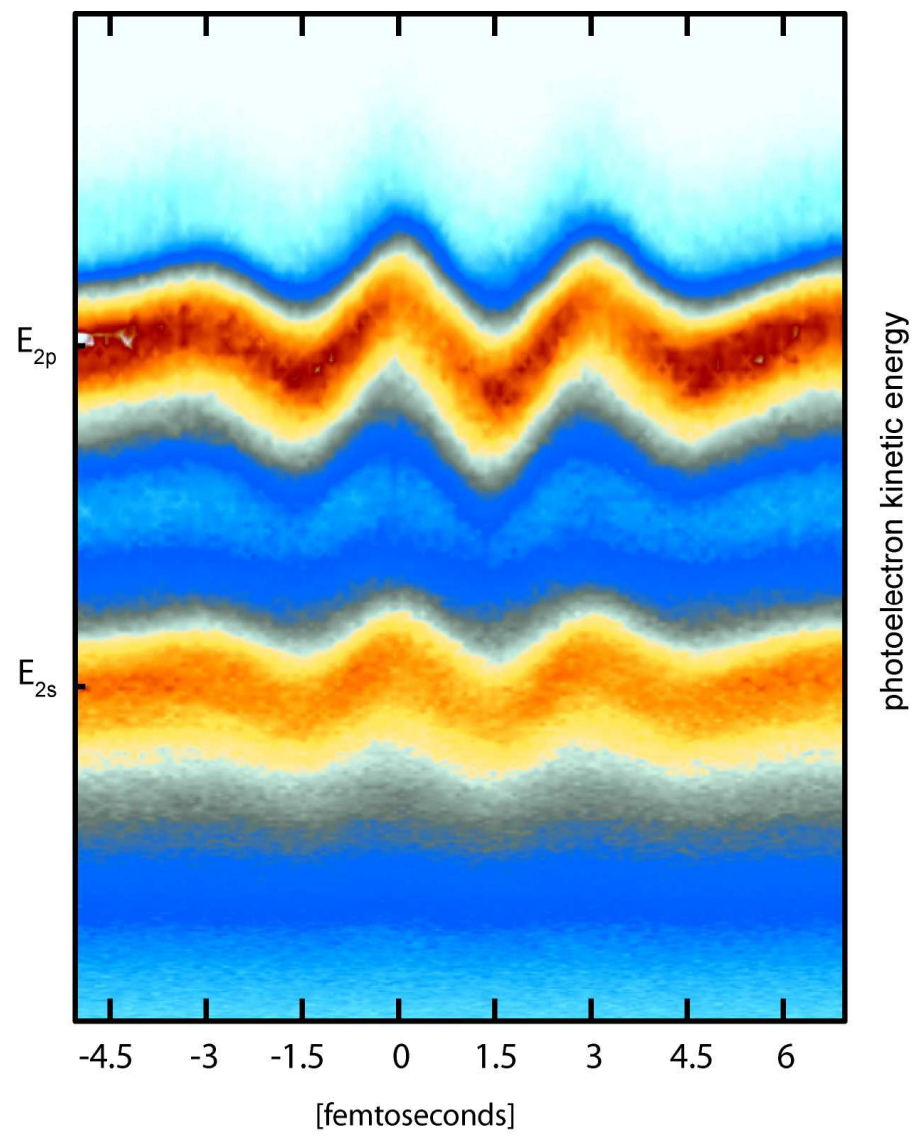

Dealy between "pump" and "probe" pulse

Fig. 5. Attosecond Streaking

If an attosecond XUV pulse releases photoelectrons from an atom while at the same time a strong visible laser pulse shines on the sample a streaking spectrogram can be recorded. When leaving the atom, the photoelectrons have a characteristic kinetic energy that corresponds to the difference between the electron affinity and the XUV photon energy. In the presence of an ultrastrong laser pulse this kinetic energy is modified by the electric field (as an electron is accelerated towards the positively charged plate in a capacitor). Since the electric field oscillates the change in kinetic energy depends on the difference in arrival time of the two pulses involved. The figure shows a measurement where the photoelectrons kinetic energy spectrum (along the vertical axis) is recorded for about 100 different delay settings between the two pulses (along the horizontal axis, the delay is given in femtoseconds). The result is an "oscilloscope" recording of the electric field of the laser pulse. Here Neon gas was ionized by an attosecond XUV pulse with photon energy about $120 \mathrm{eV}$ (corresponding to $10 \mathrm{~nm}$ wavelength) and a duration of 200 attoseconds. The photon energy is sufficient to release electrons form either the $2 p$ or the $2 s$ shell of Neon thus the measurement allows to investigate potential timing differences in in the electron emission (M Schultze et al. 2010) . 
With a step size roughly matching the XUV pulse duration the resulting spectrogram can look as in Fig. 5 and it is immediately evident that this so called "streaking spectroscopy" reveals the temporal evolution of the laser electric field (more precisely its vector potential) (E Goulielmakis et al. 2004) (Fiess et al. 2010). Beyond that, this sort of spectrograms resembles the signal of frequency resolved optical gating devices (FROG) known from laser science (Trebino \& Kane 1993); (Mairesse \& Quéré 2005); (Justin Gagnon \& Vladislav S. Yakovlev 2009). Since their origin is a cross-correlation of two unknown pulses recorded with a known response function (here the photoionization) adaptive algorithms can extract all relevant parameters of both pulses involved (Michael Hofstetter et al. 2011). Such algorithms identify the temporal structure of the attosecond pulses and their temporal and spectral phase and thus fully characterize these light bursts that are the shortest signals that can be synthesized in the laboratory. It is an interesting feature of this streaking concept that the reconstruction actually explores the temporal structure of the released photoelectron wavepacket rather than of the light pulse and thus gives access to characteristics of the photo emission process that so far remained inaccessible to experimental physics. For photon energies that are sufficiently high to address two or more individual atomic orbitals and thus liberate electrons from different energetic states (as shown in Fig. 5) the streaking technique facilitates the investigation of subtle timing differences between them. The growing ability to shape the XUV emission according to the requirements of a particular experiment extends the technique to a wider spectral range. With specially tailored attosecond XUV pulses it was possible to discover that the emission of electrons from the neon $2 \mathrm{~s}$ shell precedes the liberation of electrons originally bound in the $2 \mathrm{p}$ shell by about 20 attoseconds, the shortest time interval ever sampled directly (M Schultze et al. 2010).

\section{Conclusion}

Attosecond pulses in general allow taking snapshots of the fastest processes known in the microcosm. The permitted to resolve processes, so far only predicted, in real time and facilitated the discovery of ultra-fast mechanisms that are not yet fully understood regarding their physical origin.

The quantum mechanical tunnel effect belongs to the first category. As an implementation of attosecond tunneling spectroscopy a first experiment demonstrated how adding attosecond tools to ion mass spectrometry can shine -time-resolved- light on very fundamental processes. According to the explanation of Leonid Keldysh the probability for tunnel ionization is high at the maxima of the laser electric field und thus leads to a stepwise evolution of the number of ionized atoms exposed to intense light (Mishima et al. 2002) at the same time the time structure of subsequent Auger decay cascades in Xenon and Krypton have been resolved (Uiberacker et al. 2007). So far only known from (indirect) line width measurements, attosecond pulses turned out to be the only tool that allows unraveling complex and intertwined processes in real time.

The investigation of transport effects of electrons in a solid is an example for the second category(A. L. Cavalieri, Müller, et al. 2007). A timing difference of electrons reaching the surface after photoionization was discovered that depends on the initial state of the released electron. It was found that electrons originating from the $4 \mathrm{f}$ band of a tungsten crystal take about 50 attoseconds longer to reach the surface compared to those starting in the conduction band. Is that solely a transport effect or signature of the crystal band structure? 
Recently attosecond science was successfully combined with transient absorption spectroscopy. This experimental approach, that revolutionized quantum chemistry after the advent of pulsed laser sources, was used to track the oscillations of a coherent superposition in the valence shell of atoms in real time (Eleftherios Goulielmakis et al. 2010).

\section{References}

Anon, 1994. Femtochemistry: ultrafast dynamics of the chemical bond, Available at: http:/ / books.google.com/books?hl=de\&lr=\&id=T8IZ1aa_FRkC\&pgis=1 [Accessed October 14, 2011].

Baeva, T., Gordienko, S. \& Pukhov, A., 2006. Relativistic plasma control for single attosecond x-ray burst generation. Physical Review E, 74(6). Available at: http://pre.aps.org/abstract/PRE/v74/i6/e065401 [Accessed October 6, 2011].

Bellini, M., 2000. Generation of widely tunable harmonic pulses in the UV and VUV from a NIR optical parametric amplifier. Applied Physics B Lasers and Optics, 70(6), pp.773776. Available at:

http://www.mendeley.com/research/generation-widely-tunable-harmonicpulses-uv-vuv-nir-optical-parametric-amplifier/ [Accessed October 14, 2011].

Bulanov, S.V., Naumova, N.M. \& Pegoraro, F., 1994. Interaction of an ultrashort, relativistically strong laser pulse with an overdense plasma. Physics of Plasmas, 1(3), p.745. Available at: http:/ /link.aip.org/link/?PHPAEN/1/745/1 [Accessed October 14, 2011].

Cavalieri, A. L., Müller, N., et al., 2007. Attosecond spectroscopy in condensed matter. Nature, 449(7165), pp.1029-32. Available at: http:/ / dx.doi.org/10.1038/nature06229 [Accessed July 19, 2011].

Cavalieri, A. L., Goulielmakis, E., et al., 2007. Intense 1.5-cycle near infrared laser waveforms and their use for the generation of ultra-broadband soft-x-ray harmonic continua. New Journal of Physics, 9(7), pp.242-242. Available at:

http:/ / stacks.iop.org/1367-

2630/9/i=7/a=242?key=crossref.c8853a078987c15542c906c0692977b1

[Accessed June 29, 2011].

Christov, I., Kapteyn, H. \& Murnane, M., 1998. Dispersion-controlled hollow core fiber for phase matched harmonic generation. Optics Express, 3(10), p.360. Available at:

http:/ / www.opticsexpress.org/abstract.cfm?URI=oe-3-10-360

[Accessed October 14, 2011].

Christov, I., Murnane, M. \& Kapteyn, H., 1997. High-Harmonic Generation of Attosecond Pulses in the "Single-Cycle" Regime. Physical Review Letters, 78(7), pp.1251-1254. Available at: http://prl.aps.org/abstract/PRL/v78/i7/p1251_1 [Accessed August 3, 2011].

Ferray, M. et al., 1988. Multiple-harmonic conversion of $1064 \mathrm{~nm}$ radiation in rare gases. Journal of Physics B: Atomic, Molecular and Optical Physics, 21(3), p.L31-L35. Available at: http://stacks.iop.org/0953-4075/21/i=3/a=001 [Accessed July 27, 2011].

Fiess, M. et al., 2010. Versatile apparatus for attosecond metrology and spectroscopy. The Review of scientific instruments, 81(9), p.093103. Available at:

http://www.ncbi.nlm.nih.gov/pubmed/20886972 [Accessed September 19, 2011].

Fuji, T. et al., 2006. Parametric amplification of few-cycle carrier-envelope phase-stable pulses at $2.1 \mu \mathrm{m}$. Optics Letters, 31(8), p.1103. Available at:

http://ol.osa.org/abstract.cfm?URI=ol-31-8-1103 [Accessed October 14, 2011]. 
Gagnon, Justin \& Yakovlev, Vladislav S., 2009. The robustness of attosecond streaking measurements. Optics Express, 17(20), p.17678. Available at:

http:/ / www.opticsexpress.org/abstract.cfm?URI=oe-17-20-17678

[Accessed October 7, 2011].

Gordienko, S. et al., 2004. Relativistic Doppler Effect: Universal Spectra and Zeptosecond Pulses. Physical Review Letters, 93(11). Available at:

http:// prl.aps.org/abstract/PRL/v93/i11/e115002 [Accessed July 21, 2011].

Goulielmakis, E et al., 2004. Direct measurement of light waves. Science (New York, N.Y.), 305(5688), pp.1267-9. Available at:

http:/ / www.sciencemag.org/content/305/5688/1267.abstract

[Accessed October 6, 2011].

Goulielmakis, Eleftherios et al., 2010. Real-time observation of valence electron motion. Nature, 466(7307), pp.739-43. Available at: http://dx.doi.org/10.1038/nature09212 [Accessed July 18, 2011].

Hentschel, M. et al., 2001. Attosecond metrology. Nature, 414(6863), pp.509-13. Available at: http://dx.doi.org/10.1038/35107000 [Accessed October 7, 2011].

Hofstetter, Michael et al., 2011. Attosecond dispersion control by extreme ultraviolet multilayer mirrors. Optics Express, 19(3), p.1767. Available at:

http:/ / www.opticsexpress.org/abstract.cfm?URI=oe-19-3-1767

[Accessed October 14, 2011].

Itatani, J. et al., 2002. Attosecond Streak Camera. Physical Review Letters, 88(17). Available at: http://prl.aps.org/abstract/PRL/v88/i17/e173903 [Accessed August 7, 2011].

Kienberger, R. et al., 2004. Atomic transient recorder. Nature, 427(6977), pp.817-21. Available at: http://dx.doi.org/10.1038/nature02277 [Accessed August 11, 2011].

Kienberger, R et al., 2002. Steering attosecond electron wave packets with light. Science (New York, N.Y.), 297(5584), pp.1144-8. Available at:

http://www.sciencemag.org/content/297/5584/1144.abstract

[Accessed July 18, 2011].

Kitzler, Markus et al., 2002. Quantum Theory of Attosecond XUV Pulse Measurement by Laser Dressed Photoionization. Physical Review Letters, 88(17). Available at: http://prl.aps.org/abstract/PRL/v88/i17/e173904 [Accessed August 7, 2011].

Krausz, Ferenc, 2009. Attosecond physics. Reviews of Modern Physics, 81(1), pp.163-234. Available at: http://rmp.aps.org/abstract/RMP/v81/i1/p163_1

[Accessed July 10, 2011].

Lichters, R., Meyer-ter-Vehn, J. \& Pukhov, A., 1996. Short-pulse laser harmonics from oscillating plasma surfaces driven at relativistic intensity. Physics of Plasmas, 3(9), p.3425. Available at: http://link.aip.org/link/?PHPAEN/3/3425/1 [Accessed October 14, 2011].

L'Huillier, A. \& Balcou, Ph., 1993. High-order harmonic generation in rare gases with a 1-ps 1053-nm laser. Physical Review Letters, 70(6), pp.774-777. Available at:

http://prl.aps.org/abstract/PRL/v70/i6/p774_1 [Accessed October 14, 2011].

Mairesse, Y. \& Quéré, F., 2005. Frequency-resolved optical gating for complete reconstruction of attosecond bursts. Physical Review A, 71(1). Available at: http://pra.aps.org/abstract/PRA/v71/i1/e011401 [Accessed August 7, 2011]. 
McPherson, A. et al., 1987. Studies of multiphoton production of vacuum-ultraviolet radiation in the rare gases. Journal of the Optical Society of America B, 4(4), p.595. Available at: http:/ /josab.osa.org/abstract.cfm?URI=josab-4-4-595 [Accessed July 27, 2011].

Mishima, K. et al., 2002. Generalization of Keldysh's theory. Physical Review A, 66(3). Available at: http://pra.aps.org/abstract/PRA/v66/i3/e033401 [Accessed October 14, 2011].

Naumova, N.M. et al., 2004. Relativistic Generation of Isolated Attosecond Pulses in a $\lambda^{\wedge}\{3\}$ Focal Volume. Physical Review Letters, 92(6). Available at: http://prl.aps.org/abstract/PRL/v92/i6/e063902 [Accessed October 14, 2011].

Niikura, H. et al., 2002. Sub-laser-cycle electron pulses for probing molecular dynamics. Nature, 417(6892), pp.917-22. Available at: http://dx.doi.org/10.1038/nature00787 [Accessed June 13, 2011].

Niikura, H. et al., 2003. Probing molecular dynamics with attosecond resolution using correlated wave packet pairs. Nature, 421(6925), pp.826-9. Available at: http://dx.doi.org/10.1038/nature01430 [Accessed July 23, 2011].

Paul, P.M. et al., 2001. Observation of a train of attosecond pulses from high harmonic generation. Science (New York, N.Y.), 292(5522), pp.1689-92. Available at: http:/ / www.sciencemag.org/content/292/5522/1689.abstract [Accessed August 7, 2011].

Reichert, J., 1999. Measuring the frequency of light with mode-locked lasers. Optics Communications, 172(1-6), pp.59-68. Available at: http://dx.doi.org/10.1016/S00304018(99)00491-5 [Accessed October 14, 2011].

Strickland, D. \& Mourou, G., 1985. Compression of amplified chirped optical pulses 2 . Optics Communications, 56(3), pp.219-221. Available at: http:/ / dx.doi.org/10.1016/0030-4018(85)90120-8 [Accessed June 20, 2011].

Schultze, M et al., 2010. Delay in photoemission. Science (New York, N.Y.), 328(5986), pp.165862.

Schultze, M. et al., 2011. State-of-the-art attosecond metrology. Journal of Electron Spectroscopy and Related Phenomena, 184(3-6), pp.68-77. Available at: http:/ / dx.doi.org/10.1016/j.elspec.2011.01.003 [Accessed July 18, 2011].

Seres, J. et al., 2005. Laser technology: source of coherent kiloelectronvolt X-rays. Nature, 433(7026), p.596. Available at: http://dx.doi.org/10.1038/433596a [Accessed August 3, 2011].

Shan, B. \& Chang, Z., 2001. Dramatic extension of the high-order harmonic cutoff by using a long-wavelength driving field. Physical Review A, 65(1). Available at: http:// pra.aps.org/abstract/PRA/v65/i1/e011804 [Accessed July 4, 2011].

Sheehy, B. et al., 1999. High Harmonic Generation at Long Wavelengths. Physical Review Letters, 83(25), pp.5270-5273. Available at: http://prl.aps.org/abstract/PRL/v83/i25/p5270_1 [Accessed July 27, 2011].

Szipocs, R. et al., 1994. Chirped multilayer coatings for broadband dispersion control in femtosecond lasers. Optics Letters, 19(3), p.201. Available at: http://ol.osa.org/abstract.cfm?URI=ol-19-3-201 [Accessed September 16, 2011]. 
Telle, H.R. et al., 1999. Carrier-envelope offset phase control: A novel concept for absolute optical frequency measurement and ultrashort pulse generation. Applied Physics B: Lasers and Optics, 69(4), pp.327-332. Available at: http://www.springerlink.com/content/101jv014xejwbw6j/ [Accessed October 14, 2011].

Trebino, R. \& Kane, D.J., 1993. Using phase retrieval to measure the intensity and phase of ultrashort pulses: frequency-resolved optical gating. Journal of the Optical Society of America A, 10(5), p.1101. Available at: http:/ /josaa.osa.org/abstract.cfm?URI=josaa-10-5-1101 [Accessed October 14, 2011].

Tsakiris, G.D. et al., 2006. Route to intense single attosecond pulses. New Journal of Physics, 8(1), pp.19-19. Available at: http:/ / stacks.iop.org/1367-2630/8/i=1/a=019 [Accessed October 14, 2011].

Uiberacker, M. et al., 2007. Attosecond real-time observation of electron tunnelling in atoms. Nature, 446(7136), pp.627-32. Available at: http://dx.doi.org/10.1038/nature05648 [Accessed June 24, 2011].

Yakovlev, Vladislav S., Ivanov, Misha \& Krausz, Ferenc, 2007. Enhanced phase-matching for generation of soft $\mathrm{X}$-ray harmonics and attosecond pulses in atomic gases. Optics Express, 15(23), p.15351. Available at: http:/ / www.opticsexpress.org/abstract.cfm?URI=oe-15-23-15351 [Accessed October 14, 2011]. 


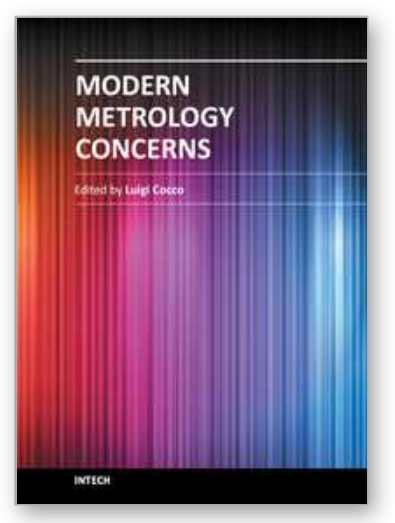

\author{
Modern Metrology Concerns \\ Edited by Dr. Luigi Cocco
}

ISBN 978-953-51-0584-8

Hard cover, 458 pages

Publisher InTech

Published online 16, May, 2012

Published in print edition May, 2012

"What are the recent developments in the field of Metrology?" International leading experts answer this question providing both state of the art presentation and a road map to the future of measurement science. The book is organized in six sections according to the areas of expertise, namely: Introduction; Length, Distance and Surface; Voltage, Current and Frequency; Optics; Time and Relativity; Biology and Medicine. Theoretical basis and applications are explained in accurate and comprehensive manner, providing a valuable reference to researchers and professionals.

\title{
How to reference
}

In order to correctly reference this scholarly work, feel free to copy and paste the following:

Martin Schultze and Reinhard Kienberger (2012). Attosecond Metrology and Spectroscopy, Modern Metrology Concerns, Dr. Luigi Cocco (Ed.), ISBN: 978-953-51-0584-8, InTech, Available from:

http://www.intechopen.com/books/modern-metrology-concerns/attosecond-metrology-and-spectroscopy

\section{INTECH}

open science | open minds

\author{
InTech Europe \\ University Campus STeP Ri \\ Slavka Krautzeka 83/A \\ 51000 Rijeka, Croatia \\ Phone: +385 (51) 770447 \\ Fax: +385 (51) 686166 \\ www.intechopen.com
}

\author{
InTech China \\ Unit 405, Office Block, Hotel Equatorial Shanghai \\ No.65, Yan An Road (West), Shanghai, 200040, China \\ 中国上海市延安西路65号上海国际贵都大饭店办公楼 405 单元 \\ Phone: +86-21-62489820 \\ Fax: $+86-21-62489821$
}


(C) 2012 The Author(s). Licensee IntechOpen. This is an open access article distributed under the terms of the Creative Commons Attribution 3.0 License, which permits unrestricted use, distribution, and reproduction in any medium, provided the original work is properly cited. 